\title{
Monitoring Change of Body Fluid during Physical Exercise using Bioimpedance Spectroscopy and Finite Element Simulations
}

\author{
Lisa Röthlingshöfer ${ }^{1}$, Mark Ulbrich ${ }^{1}$, Sebastian Hahne ${ }^{1}$, Steffen Leonhardt ${ }^{1}$ \\ 1. Philips Chair for Medical Information Technology, RWTH Aachen University, 52074 Aachen, Germany \\ E-mail any correspondence to: roethlingshoefer@hia.rwth-aachen.de or ulbrich@hia.rwth-aachen.de
}

\begin{abstract}
Athletes need a balanced body composition in order to achieve maximum performance. Especially dehydration reduces power and endurance during physical exercise. Monitoring the body composition, with a focus on body fluid, may help to avoid reduction in performance and other health problems. For this, a potential measurement method is bioimpedance spectroscopy (BIS). BIS is a simple, non-invasive measurement method that allows to determine different body compartments (body fluid, fat, fat-free mass). However, because many physiological changes occur during physical exercise that can influence impedance measurements and distort results, it cannot be assumed that the BIS data are related to body fluid loss alone. To confirm that BIS can detect body fluid loss due to physical exercise, finite element (FE) simulations were done. Besides impedance, also the current density contribution during a BIS measurement was modeled to evaluate the influence of certain tissues on BIS measurements. Simulations were done using CST EM Studio (Computer Simulation Technology, Germany) and the Visible Human Data Set (National Library of Medicine, USA). In addition to the simulations, BIS measurements were also made on athletes. Comparison between the measured bioimpedance data and simulation data, as well as body weight loss during sport, indicates that BIS measurements are sensitive enough to monitor body fluid loss during physical exercise.
\end{abstract}

Keywords: Bioimpedance Spectroscopy, dehydration, FE simulation, physical exercise

\section{Introduction}

The human body consists of different compartments such as bones, fat and body water. A balance between all these compartments is essential for a person's health, quality of life and sport performance. Especially body water (that accounts for $50-70 \%$ of total body weight) is important with regard to physical exercise and endurance. Sawka showed that athletes and workers frequently dehydrate by 3-6\%, which decreases their cognitive and physical performance [1]. Also, during one game, soccer players have a loss of body weight of 1-2.5 $\mathrm{kg}$ [2] or of 2-4 1 body water [3, 4]. In all cases, the amount of body water loss depends on ambient temperature, humidity, length of exercise, nutritional intake, clothing, airflow and heat acclimatization status [5]. A considerable loss of body weight and body fluid may cause dehydration with severe consequences. Dehydration reduces central blood volume and skin blood flow. Furthermore, decreased blood volume causes an increase in heart rate, thus reducing stroke volume [5]. Finally, dehydration can re- sult in loss of motion control, confusion, nausea and even death [6]. To avoid these consequences, the monitoring of body composition is important. Bioimpedance spectroscopy (BIS) allows such monitoring in a non-invasive, rapid and inexpensive way. Compared with other measurement methods, BIS provides a good estimation of water content during changes in hydration status $[6,7]$. However, to obtain precise BIS measurements controlled conditions are required to avoid the influence of external factors such as electrode placement, subject's posture and/or body movements [9]. In relation to physical exercise, several other factors also need to be considered. One such factor is temperature, because both body and skin temperature affect body impedance. Cornish et al. investigated the influence of temperature on skin impedance and showed that skin resistance and reactance decreases by $35 \%$ and $18 \%$, respectively, in a temperature range of $20{ }^{\circ} \mathrm{C}-40{ }^{\circ} \mathrm{C}$ [10]. Representing body fluids as electrolyte solution, Grimnes et al. reported an impedance change of $+2 \% /{ }^{\circ} \mathrm{C}$ [11]. Heat stress combined with physical exercise also alters fluid distribution between vascular, interstitial and intracellular compartments [12]. In addition, sweat is produced, which results in improved skin conductivity and therefore reduced skin impedance. Sawka and Coyle described sweat rates of 1-2 1/h during vigorous exercise in the heat [13]. Finally, physical exercise leads to increased vascular perfusion and cutaneous blood flow, hyperemia and vasodilatation [14]. O'Brien et al. reported that all these factors influence overall body impedance and can complicate the detection of body fluid loss [15]. They analyzed several studies and found that impedance change was not uniform. Although only athletes were measured, in some studies body impedance increased during training whereas in other studies impedance decreased [15, 16, 17, 18, 19]. These differences emphasize that more studies are needed to clarify whether BIS measurements are sensitive enough to accurately detect body fluid loss during physical exercise. One possible approach is to use simulations based on physical models. Finite element (FE) simulations are often used to elucidate processes and measurement setups, and to analyze influencing factors. However, simulating measurements on a human body is difficult due to the specific properties of the human body (e.g. magnetic permittivity, electric conductivity/permittivity) which are not well described and depend on a person's activity [20]. Therefore, only a few groups have simulated impedance measurements on a human body. For example, Beckmann et al. used simulations to optimize electrode placement for thoracic BIS measure- 


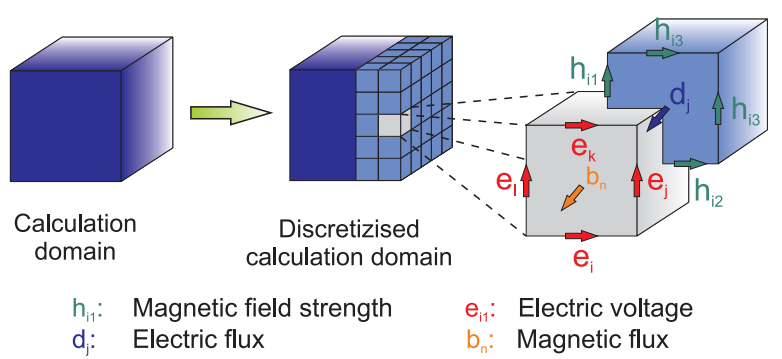

Fig. 1: Discretization of electromagnetic properties to a dual FIT grid [25]

ments [21]. Wang et al. reported that FE models provide good information on optimal electrode positions and sources for thoracic impedance changes in impedance cardiography [22]. Gagnon et al. developed an FE model to simulate the electrical characteristics of benign and malignant cells to better understand skin impedance measurements [23]. Finally, Barchanski et al. tried to reproduce a whole body BIS measurement and compare it to real measurements [24].

\section{Technical Basics}

\section{Finite Integration Technique (FIT)}

FE simulations of BIS measurements are used to model the conductivity and permittivity contribution of different tissues in the human body to determine certain measurement scenarios and influences. Therefore, complex geometries are divided into simple finite elements. These elements can be rectangles or triangles for $2 \mathrm{D}$ problems, and pentahedrons, pyramids, tetrahedrons or hexahedrons for 3D problems. Physical values are assigned to the edges, faces and volumes of these substructures. Figure 1 shows the discretization of the electromagnetic properties.

Using the FIT, magnetic (b) and electric fluxes (d) are allocated on the faces of the grids while electric voltages (e) and magnetic field strength (h) are assigned to the edges. Hence, a system of equations, called Maxwell-Grid Equations, has to be solved for the whole calculation domain, where each cell is described by:

$$
\begin{array}{ll}
C \vec{e}=-\frac{\partial \vec{b}}{\partial t} & \tilde{C} \vec{h}=\frac{\partial \vec{d}}{\partial t}+\vec{j} \\
\tilde{S} \vec{d}=q & S \vec{b}=0
\end{array}
$$

Note that both $\mathrm{C}$ matrices correspond to the curl operator and both $\mathrm{S}$ matrices to the divergence operator [26]. These equations can be simplified for this work because of electroquasistatic conditions (see chapter Methods).

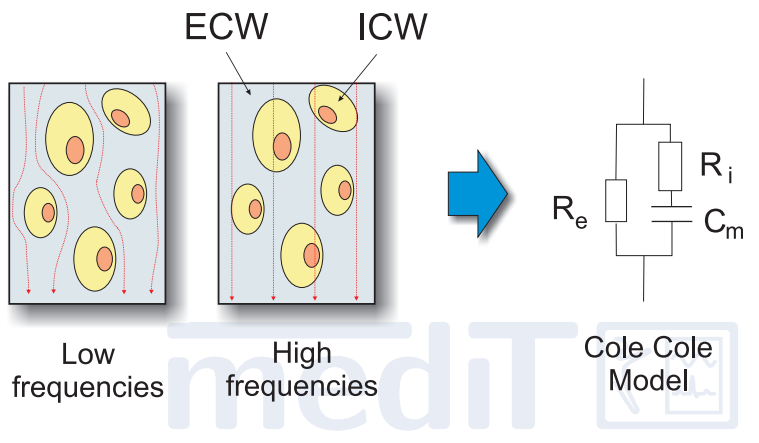

Fig. 2: Current flow in tissue and electrical equivalent circuit

\section{Bioimpedance Spectroscopy Technique}

During a BIS measurement, four electrodes are attached to the body: two outer electrodes inject $\mathrm{AC}$ currents usually with frequencies between $5 \mathrm{kHz}$ and $1 \mathrm{MHz}$ and the other two measure the resulting voltage. Knowing voltage and current, body impedance can be calculated. To determine water content, the electrical characteristics of human tissue must be taken into account. Various body compartments, e.g. blood and muscle, have higher conductivities compared with, e.g., bone and fat [10]. According to the relative amount of each tissue and body fluid, the electrical characteristics of the human body change. Body fluid itself can be divided into extracellular water (ECW) and intracellular water (ICW), representing extra- and intracellular volume (ECV, ICV) (see figure 2).

These volumes are separated by the cellular membrane. ECW and ICW are predominantly electrically resistive entities $\left(R_{e}, R_{i}\right)$, whereas the cellular membrane, due to its lipid bilayer, has a capacitive behavior $\left(C_{m}\right)$. Accordingly, the distribution of an injected current depends on its frequency: a low frequency current flows only around the cells, whereas a high frequency current will pass through the cell membrane and the ICW. Such behavior can be represented by an electrical model, known as the Cole model [27], and described by the following equation:

$$
Z(j \cdot \omega)=\frac{R_{e}}{R_{e}+R_{i}} \cdot\left(R_{i}+\frac{R_{e}}{1+\left(j \omega C_{m}\left(R_{e}+R_{i}\right)\right)^{\alpha}}\right) \cdot e^{-j \omega T_{d}}
$$

In this equation, $\alpha$ is a heuristic factor representing the presence of different tissues in parallel with specific time constants. $T_{d}$ is the dead time produced by e.g. the stray capacities of the measurement device. $R_{e}$ and $R_{i}$ are the extra- and intracellular resistance and $C_{m}$ the membrane capacity [28]. Using the Cole parameters and the basics of the Hanai Theory [29], equations for the calculation of ECW, ICW, and total body water have been provided [30].

$$
E C V=\frac{\rho_{e}^{\frac{2}{3}}}{3 \cdot(4 \pi)^{\frac{1}{3}} \cdot 1000}\left(\frac{l}{C_{1} \cdot C_{2} \cdot R_{e}}\right)^{\frac{2}{3}} \cdot l \cdot\left(C_{1}^{2}+C_{2}^{2}+C_{1} C_{2}\right)
$$




$$
I C V=E C V\left(\left[\frac{\left(\rho_{i}-\left(\rho_{i}-\rho_{e}\right)\right) R_{0}}{\rho_{e} \cdot R_{i n f}}\right]^{\frac{2}{3}}-1\right)
$$

These two equations approximate all body segments as frustums with the length 1 and the circumferences $C_{1}$ and $C_{2}$. Here, $\rho_{e}=40.3 \Omega \mathrm{cm}$ and $\rho_{i}=153.95 \Omega \mathrm{cm}$ are empirically assessed values. To calculate the fat free mass (FFM), the following equation can be used [28]:

$$
F F M=\left(d_{e} \cdot E C V\right)+\left(d_{i} \cdot I C V\right)
$$

Here, $d_{e}=1.106$ is the mean density of ECW and $d_{i}=1.521$ the mean density of ICW. The muscle mass (MM) is calculated by an empirically assessed equation [31]:

$$
M M=\frac{I Z V-0.127 \cdot F F M \cdot 0.068}{0.5545}
$$

Using this $\mathrm{MM}$ and the real part of the measured impedance, the muscle conductivity for $f \rightarrow \infty$ and $f \rightarrow 0$ can be calculated.

\section{Methods}

\section{The Simulation Model}

BIS measurements were simulated using an anatomical data set of a male human. The data set is based on the Visible Human Data Set (National Library of Medicine, MD, USA) providing resolutions from $1 \times 1 \times 1 \mathrm{~mm}^{3}$ to $8 \times 8 \times 8 \mathrm{~mm}^{3}$ [32]. The source of this data is an executed prisoner, Joseph Paul Jernigan, who was frozen into gelatin after death. His frozen body has been cut into more than 1800 slices, socalled cryosections. These slices have been digitized using MRI and CT with an image resolution of $4096 \times 2700$ pixels and a 24 bit color depth. For the presented simulation model, an anatomical data set created by MVR Studio GmbH (Loerrach, Germany) was used.

This data set already contains segmented voxels for all important tissues. Since voxels contain no volume data for a certain tissue, the voxel data had to be converted into volumes. First, all tissues were converted into stereolithography files containing surface geometries composed of triangles, represented by polygon meshes, enclosing the different tissues. This enclosed area can be converted into volume data, which was done for every type of tissue. Using Boolean operations, all tissue volumes were combined to form the whole body. Therefore, one volume containing all tissues was created using fatty tissue as a meta structure into which all other tissues were inserted by subtracting them from this meta tissue. Since the highest resolution $\left(8 \times 8 \times 8 \mathrm{~mm}^{3}\right)$ was used, the model contained no skin tissue. Thus, a new skin layer was added beyond the electrodes. The thickness of this additional skin layer was $5 \mathrm{~mm}$ (which is significantly thicker than real human skin). Unfortunately, due to the model resolution and the cylindrical body shape, thinner skin could not be generated. The BIS electrodes composed of aluminum and PEC (perfect electric conducting) layers were added on both feet which corresponds to a leg-to-leg BIS measurement. The resulting volume model consumed 3.3 GB RAM.

Conductivity $(\sigma)$ and permittivity $\left(\varepsilon_{r}\right)$ values for every tissue have been implemented using the data from Gabriel et al. (see table 1) [27].

Tab. 1: Permittivity and conductivity values at $100 \mathrm{kHz}$ [27]

\begin{tabular}{lcc}
\hline & $\sigma[\mathrm{S} / \mathrm{m}]$ & $\varepsilon_{r}$ \\
\hline Skin & 0.065836 & 15357 \\
Bone & 0.020791 & 227.64 \\
Fat & 0.024414 & 92.885 \\
Muscle & 0.36185 & 8089.2 \\
\hline
\end{tabular}

For the simulation the PECs were charged with a fixed voltage. To calculate the complex current, the current density was integrated over transversal faces. Since the maximum wavelength is much higher than our model volume, the low frequency electroquasistatic solver with the following boundary conditions was used for the simulation:

$$
\frac{\partial \vec{D}}{\partial t}=0 \text { and } \frac{\partial \vec{B}}{\partial t}=0
$$

Due to these boundary conditions, the complex current cannot be determined directly. Assuming $\vec{D}$ as a harmonic oscillation $\left(\vec{D}=|\vec{D}| \cdot e^{j \omega t} \cdot \vec{e}_{x}\right)$ to access the displacement current, the complex current could be calculated using the following equation:

$$
\underline{I}=\int_{A}\left(\vec{J}+\frac{\partial \vec{D}}{\partial t}\right) \cdot d \vec{A}=\int_{A}(\vec{J}+j \omega \vec{D}) \cdot d \vec{A}
$$

The simulation of an ankle-to-ankle BIS measurement with a mesh density of 2 million tetrahedrons consumed 5 GB RAM and took 25 hours per sweep. The calculation frequency ranged from $5 \mathrm{kHz}$ to $5 \mathrm{MHz}$, covering the measuring range of common BIS devices, which usually is $5 \mathrm{kHz}$ to $1 \mathrm{MHz}$. The slightly bigger frequency range ensures the simulation of preferably complete Cole curves. Matlab (The Mathworks Inc., USA) was used to analyze impedances obtained by the simulations to compute $R_{e}$ and $R_{i}$.

\section{Bioimpedance Spectroscopy Technique}

To validate and compare the simulation results, real BIS measurements were made in a group of athletes. The aim was to test the sensitivity of real measurements to detect body fluid loss during physical training. In this trial, the hydration level of the 10 test subjects (aged 22-34 years; Table 2) was monitored during exercise on a cross trainer (Ergo Lyps Medical, Daum Electronics, Fürth, Germany). As reported earlier, it is difficult to measure changes in impedance during physical exercise and results are not always uniform [14]. Because skin temperature plays a major role, the infrared VarioCAM hr head camera (Jenoptik, Germany) was used to define the time range in which skin temperature is constant 
Tab. 2: Physical data of the ten athletes.

\begin{tabular}{lllll}
\hline $\begin{array}{l}\text { Athlete's } \\
\text { ID no. }\end{array}$ & $\begin{array}{l}\text { Age } \\
\text { (years) }\end{array}$ & $\begin{array}{l}\text { Height } \\
(\mathrm{m})\end{array}$ & $\begin{array}{l}\text { Weight } \\
(\mathrm{kg})\end{array}$ & Sex \\
\hline 1 & 27 & 1.99 & 101.4 & Male \\
2 & 22 & 1.89 & 75.5 & Male \\
3 & 27 & 1.78 & 60.8 & Male \\
4 & 31 & 1.71 & 81.5 & Male \\
5 & 34 & 1.70 & 76.9 & Male \\
6 & 26 & 1.70 & 69.3 & Female \\
7 & 25 & 1.77 & 71.8 & Female \\
8 & 25 & 1.89 & 75.6 & Male \\
9 & 25 & 1.78 & 80.7 & Male \\
10 & 25 & 1.71 & 74.9 & Male \\
\hline
\end{tabular}

during exercise. During this period, body fluid loss can be analyzed using BIS.

The ten athletes were requested to work out on the cross trainer for specific time periods (warm up time $+3 \times 30 \mathrm{~min}$ training + cooling down time). In the defined periods, whole body BIS measurements (wrist-to-ankle) were made with a Hydra 4200 (Xitron Technologies, USA) as reference measurements since this is the standard measurement method [7]. In five athletes, ankle-to-ankle impedance as a new measurement method was also measured and analyzed in order to compare measurements made in athletes with the simulation data. Ankle-to-ankle BIS measurements or even thigh-tothigh measurements have some advantages over whole body measurements. Investigations of Medrano and Beckmann et al. have shown that these measurement techniques are more suitable focusing on wearable BIS applications, which are needed for continous sport monitoring [8]. In addition, images of the legs and arms were made with the infrared camera. Athletes did not drink or eat for $2 \mathrm{~h}$ before and during measurements to ensure a controlled fluid loss. As a reference for dehydration, each athlete was weighed before and after training, and the temperature in the gym room was kept constant. To exclude influences of body fluid shifts, all subjects had to lie down for a certain time before measurements were made and every measurement was made with exactly the same body posture [9].

\section{Results}

\section{Simulation Results}

For the simulation of dehydration during physical exercise, several assumptions were made. Dehydration describes the loss of total body water, which increases whole body impedance. Since muscle is one of the main water reservoirs in the human body, dehydration during physical exercise leads to fluid loss mainly in this tissue. As a consequence, muscle conductivity decreases. According to these assumptions, muscle conductivity of the visible human model was reduced for the simulation of dehydration. Since no conductivity values for dehydrated muscle tissue are available, these

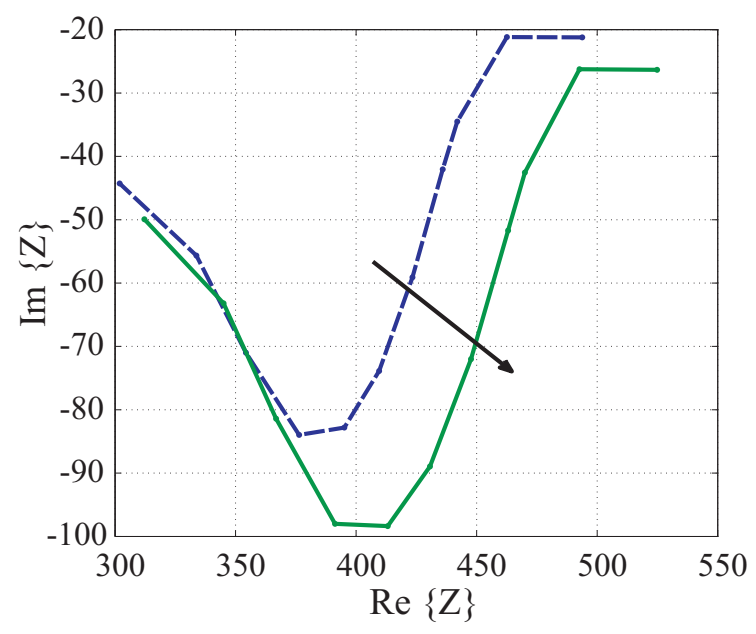

Fig. 3: Simulated dehydration of the legs

were assessed by measurements with the Xitron Hydra on additional athletes (not the validation group for the simulation) and for all athletes the MM was calculated before and after exercise according to equation 7 to determine the change of muscle tissue conductivity $(\approx 0.045 \mathrm{~S} / \mathrm{m})$. The results of the simulations are shown in figure 3.

The simulation showed a clear impedance shift to higher values $(6 \%)$. This implies that the change of muscle conductivity is visible in the impedance measurements. Consequently, it can be assumed that body fluid loss during physical exercise can be detected using BIS. Additional confirmation of these results is provided by analysis of the current density contribution. For this, three cutting planes were defined in the middle of the thigh, the knee and the lower leg. At each cutting plane, the frequency-dependent current density was calculated for each tissue. Table 3 presents the current density contribution.

Tab. 3: Current density contribution (in percentage) at $1 \mathrm{MHz}$.

\begin{tabular}{llll}
\hline Tissue/Cutting Plane & Thigh & Knee & Lower Leg \\
\hline Bone & $0.1 \%$ & $2.07 \%$ & $0.27 \%$ \\
Muscle & $96.05 \%$ & $84.82 \%$ & $98.95 \%$ \\
Fat & $3.86 \%$ & $13.12 \%$ & $0.77 \%$ \\
\hline
\end{tabular}

The results show that the main current flowed through muscle $(84.82 \% ; 98.95 \%)$. This can be explained by good perfusion and high water content in muscle, resulting in good muscle conductivity $(0.32-0.5 \mathrm{~S} / \mathrm{m})$. The high current density in muscle tissue compared to other tissue confirms the considerable influence of muscle tissue on BIS measurements. Thus, BIS measurements are highly sensitive to changes in the electrical properties of muscle tissue and, consequently, to loss of body fluid during physical exercise. Even a small variation will lead to a large impedance shift. However, the simulation showed one deviation. In leg, the values of simulated absolute impedance are much higher than those of real measured impedance $(\approx 490 \Omega$ [33]). This difference can be due to a variety of reasons. The main factor is the FE model resolution $\left(8 \times 8 \times 8 \mathrm{~mm}^{3}\right)$ that leads to inaccuracy. During the conversion process, 

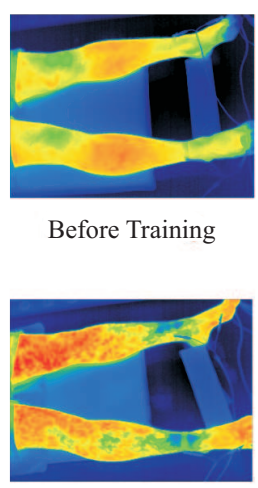

+30 min training
Before Training

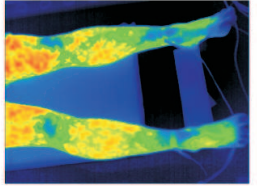

+15 min warm up

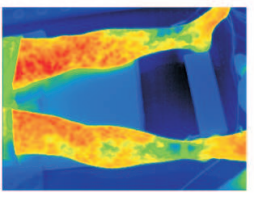

+30 min training

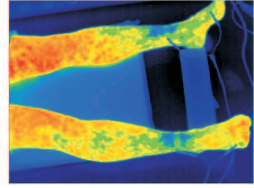

+30 min training

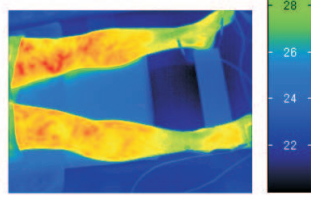

+15 min recovery

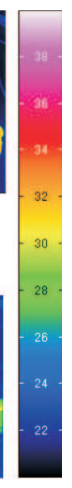

Fig. 4: Infrared images of the leg of athlete no. 5 showing his temperature profile during the trial.

small structures such as blood vessels (e.g. arterioles) were 'lost'; these volumes are too small for the model and are replaced by the meta-volume fat which has less conductivity. Another reason for higher impedance is the lack of skin in the original voxel model. The $5 \mathrm{~mm}$ skin volume that was added later on is much thicker and does not reflect the realistic skin impedance. Last but not least, a 2-point measurement was simulated instead of a 4-point measurement which is used when measuring real persons. Thus, the electrode-skin interface is part of the simulated impedance.

\section{BIS Measurement Results}

In this study, the analyses were performed in two steps. First, temperature measurements taken with the infrared camera were evaluated and time periods with stable temperature profiles were defined. Then, the Cole parameters were determined and analyzed for the specified time periods. Finally, the results of the study were compared with the simulation data.

Figure 4 presents infrared images of athlete (ID no. 5) during the trial, as an example of the temperature profile during a complete measurement. The first picture was taken before the training $(0 \mathrm{~min})$; at that moment, the athlete was relaxed and rested. The skin temperature ranges from $27{ }^{\circ} \mathrm{C}$ to $32{ }^{\circ} \mathrm{C}$. After starting to work out, skin temperature increased due to muscle activity and skin perfusion. This temperature change was visible in the lighter areas on the skin during the training period. Even after a recovery time of $15 \mathrm{~min}$, the legs were still warm and well perfused. Thus, a 15-min period is probably too short for a complete cool down, and a longer recovery period is necessary to return to the baseline conditions. This effect was observed in all athletes and can also be seen in the temperature profile of test person no. 10 (Fig. 5). The skin temperature of the last measurement was still $2{ }^{\circ} \mathrm{C}$ higher than the skin temperature at the beginning of the trial. Comparisons between the first and last impedance measurements are therefore not recommended.

Another interesting effect is visible at the beginning of the temperature profile. In some cases skin temperature did not increase immediately after the start of training. In contrast, skin temperature decreased within the first 20 min despite the

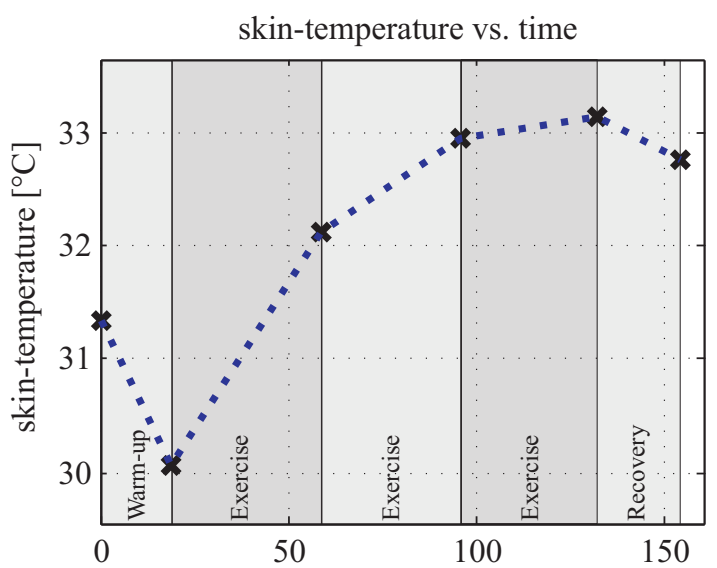

time-interval relating to first measurement [min]

Fig. 5: Temperature profile of athlete no. 10 measured on the hand.

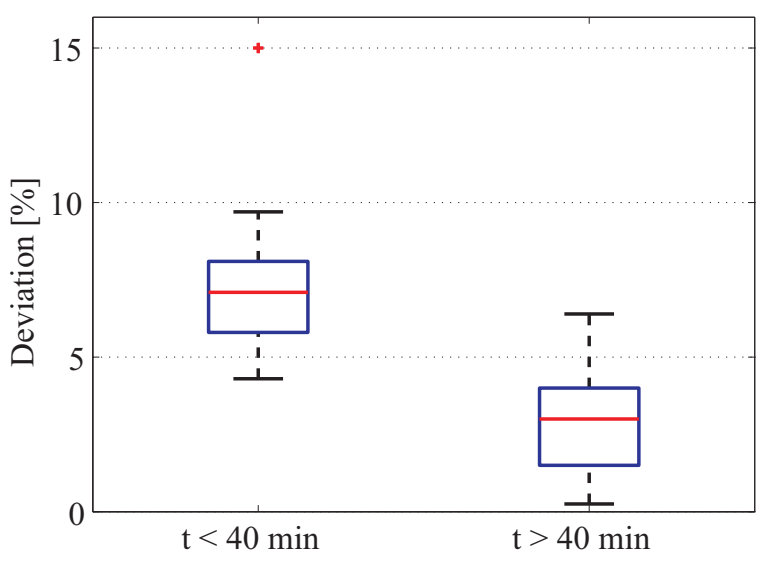

Fig. 6: Plot showing deviations in skin temperature.

warming-up period. One reason for this could be the convective heat transfer. At the start of training the body needs a certain amount of time to warm up, and to activate vascular perfusion and muscle activity, so that skin temperature increases slowly. However, the arms were already moving and heat transfer between the moving arms and surrounding air started immediately after training started. It is possible that the cooling effect prevails at the start of training, which leads to a decrease in skin temperature within the first $20 \mathrm{~min}$. Afterwards, heat production due to muscle activity and skin perfusion is dominant and skin temperature increases. Since changes in temperature have a considerable impact on BIS measurements, impedance can only be analyzed, compared and evaluated during stable temperature ranges. Therefore, the training period was divided into two parts. Figure 6 shows the temperature deviation in all athletes during the first $40 \mathrm{~min}$ and during the remainder of the training.

In the first period higher temperature changes were measurable $(\approx 7 \%)$, whereas after the first 40 min skin temperature remained stable $(\approx 3 \%)$. As a consequence, only impedance data measured after the first 40 min of the training were used to analyze the sensitivity of the BIS measurement. Based on these temperature profiles, measured impedance data were analyzed and the Cole parameters were calculated. After the first $40 \mathrm{~min}$ the extracellular resistance of all athletes increased. Table 4 shows the change in whole body 


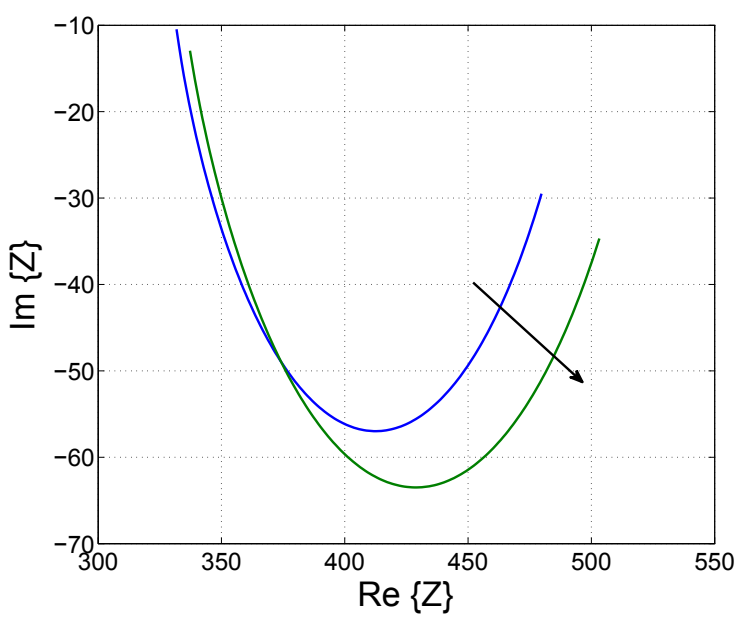

Fig. 7: Measured dehydration of the legs

$R_{e, r e l}$, as well as the change in $R_{e, A A}$ measured between ankleto-ankle for all ten athletes.

Tab. 4: Change of $R_{e}$ and weight for all 10 athletes.

\begin{tabular}{llll}
\hline Subjects & \multicolumn{3}{c}{ Parameter } \\
\hline & $\Delta R_{e, \text { rel }}[\%]$ & $\Delta R_{e, A A}[\%]$ & $\Delta$ Weight $[\%]$ \\
\hline 1 & 5.2 & 3.4 & -2.3 \\
2 & 2.8 & 1.9 & -3.0 \\
3 & 1.7 & 0.2 & -3.3 \\
4 & 0.5 & 1.4 & -2.2 \\
5 & 4.0 & 0.24 & -2.3 \\
6 & 4.21 & - & -1.2 \\
7 & 2.22 & - & -1.5 \\
8 & 2.59 & - & -1.3 \\
9 & 4.94 & - & -0.8 \\
10 & 2.46 & - & -1.3 \\
\hline
\end{tabular}

The change in $R_{e, r e l}$ of the whole body ranged from 0.5 $5.2 \%$, with an average of $3.06 \%$. The change in $R_{e, A A}$ of the ankle-to-ankle measurements ranged from $0.2-3.4 \%$, with an average of $0.7 \%$. These increases of impedance reflect the measured loss of water. In addition, comparing measurement results (figure 7) with the simulation data (figure 3), almost identical impedance shifts are observed. First, by visual inspection of these figures, one can observe an impedance shift to higher impedances indicating a fluid loss. Second, analyzing the low frequency impedance change, the simulated impedance increases about $6 \%$, and the measured impedance about $5 \%$.

In addition, a correlation coefficient near $r=1$ can be obtained comparing the static simulation with the static measurement. These findings confirm the accuracy of the simulation. Since body weight measurements before and after training ensured that each athlete lost body water during training (see table 4), it is assumed that the measured impedance shift corresponds to body fluid loss and, thus, BIS measurements are sensitive enough to detect dehydration even during real physical exercise.

\section{Conclusion}

In this work, changes of body fluid due to physical exercise were analyzed using FE simulations of BIS measurements and real measurements in athletes before, during and after training. FE simulations were based on an anatomical data set from a male human. The simulated ankle-to-ankle BIS measurements show a clear shift to higher impedances. This verifies the sensitivity of BIS to changes in muscle conductivity and, therefore, to body fluid loss during physical exercise. Comparison of the real measured impedance data and the simulation data confirm that, under controlled temperature conditions, impedance changes and body fluid loss can be detected during physical exercise. It is shown that FE simulations are a valuable tool to interpret BIS measurements by taking an insight into the human body and being able to analyze where current paths run and which changes affect measured impedances. Thus it it possible to identify sources for adapting models in the future. Additional factors that may influence BIS measurements during physical activity (e.g. changes in perfusion or ion losses) should also be investigated using FE simulations.

\section{Acknowledgements}

This work was part of the NUTRIWEAR project. This project was supported by the German Federal Ministry of Education and Research in the Microsystems program 20042009

\section{References}

1. Sawka M. N., Physiological consequences of hypohydration: exercise performance and thermoregulation, Med Sci Sports Exer, 24, 657-670 (1992).

2. Ekblom B., Applied physiology of soccer, Sports Med, 3, 50-60, (1986). http://dx.doi.org/10.2165/00007256-198603010-00005

3. Mustafa I. K. Y. and Mahmoud N. E. A., Evaporative water loss in African soccer players, J Sports Med Phys Fitn, 19, 181-183, (1979).

4. Bangsbo J., The physiology of soccer - with special reference to intense intermittent exercise, Copenhagen: University of Copenhagen, 1993.

5. Casa D. J. and Stearns R.L. and Lopez R.M.and Ganio M.S., Influence of hydration on physiological function and performance during trail running in the heat, Journal of Athletic Training, 45, 147-156, (2010). http://dx.doi.org/10.4085/1062-6050-45.2.147

6. Higgins K. and Reid P.H. and Going S.B. and Howell W.H., Validation of bioimpedance spectroscopy to assess acute changes in hydration status, Medicine \& Science in Sports \& Exercise, 984-990, (2007). http://dx.doi.org/10.1249/mss.0b013e31803bb4d4

7. Moissl U. and Wabel P. and Chamney P. et al.,Body fluid volume determination via body composition Spectroscopy in health and disease, Physiol. Measurement, 27, 921-933, (2006). http://dx.doi.org/10.1088/0967-3334/27/9/012 
8. Medrano G. and Beckmann L. and Gube M. and Kasim R. and Kim S. and Kraus T. and Leonhardt S., Continous Hand-to-Foot and Segmental Bioimpedance Spectroscopy Measurements within a Period of Five Days, World Congress on Medical Physics and Biomedical Engineering, IFMBE Proceedings, 25, 122-125, (2009). http://dx.doi.org/10.1007/978-3-642-03885-3_35

9. Medrano G. and Eitner F. and Walter M. and Leonhardt S., Model-based correction of the influence of body position on continuous segmental and hand-to-foot bioimpedance measurements, Med Biol Eng Comput, 48, 531-541 (2010). http://dx.doi.org/10.1007/s11517-010-0602-5

10. Cornish B.H. and Thomas B.J. and Ward L.C., Effect of temperature and sweating on Bioimpedance Measurements, Appl.Radiat.Isol, 49, No.5/6, 475-476, (1998). http://dx.doi.org/10.1016/S0969-8043(97)00057-2

11. Grimnes S. and Martinsen O., Bioimpedance and Bioelectricity Basics, 1st ed. Academic Press. London, (2000).

12. O'Brien C. and Young A.J. and Sawka M.N., Bioelectrical impedance to estimate changes in hydration status, Int J Sports Med, 23, 361-366, (2002). http://dx.doi.org/10.1055/s-2002-33145

13. Sawka M. N. and Coyle E. F., Influence of body water and blood volume on thermoregulation and exercise performance in the heat, Exerc Sport Sci Rev, 27, 167-218,(1999).

14. Segal K., Use of bioelectrical impedance analysis measurements as an evaluation for participating in sports, Am J Clin Nutr, 57 (suppl), 469-471, (1996).

15. O'Brien C. and Baker-Fulco C.J. and Young A.J. and Sawka M.N., Bioimpedance assessment of hypohydration, Med Sci Sports Exerc, 31, 1466-1471, (1999). http://dx.doi.org/10.1097/00005768-199910000-00017

16. Saunders M.J. and Blevins J.E. and Broeder C.E., Effect of hydration changes on bioelectrical impedance in endurance trained individuals, Med Sci Sports Exer., 30, 885-892, (1998). http://dx.doi.org/10.1097/00005768-199806000-00017

17. Khaled M.A. and McCutcheon M.J.and Reddy S.and Pearman P.L. et al., Electrical impedance in assessing human body composition: the BIA method, Am J Clin Nutr, 47, 789-792, (1988).

18. Jürimäe J. and Jürimäe T. and Pihl E., Changes in body fluids during endurance rowing training, Annals of the New York Academy of Sciences, 904, 353-358, (2000).

19. Caton J.R. and Molé P.A. and Adams W.C and Heustis D.S., Body composition analysis by bioelectrical impedance: effect of skin temperature, Med and Sci in Sports and Exerc, 20, 489-491, (1988).

20. Siauve N. and Scorretti R. and Burais N. and Nicolas L. and Nicolas A., Electromagnetic fields and human body: a new challenge for the electromagnetic field computation, Compel, 22, 457-469, (2003).

http://dx.doi.org/10.1108/03321640310474868

21. Beckmann L. and van Riesen D. and Leonhardt S., Optimal electrode placement and frequency range selection for the detection of lung water using Bioimpedance Spectroscopy, 29th Annual International Conference of the IEEE Engineering in Medicine and Biology Society - EMBC 2007, Lyon, France, (2007).

\section{http://dx.doi.org/10.1109/IEMBS.2007.4352882}

22. Wang Y. and Haynor D. R. and Kim Y., A finite-element study of the effects of electrode position on the measured impedance change in impedance cardiography, IEEE Transactions on Biomedica Engineering, 48, No.12, (2001). http://dx.doi.org/10.1109/10.966598

23. Gagnon H. and Guardo R. and Kokta V. and Hartinger A. E., A hybrid FEM model to simulate the electrical characteristics of biological tissues at the cellular level, Journal of Physics: Conference Series 224, (2010).

24. Barchanski A. and Wittorf S. and Weiland T., Simulation of bioimpedance spectroscopy using the finite integration technique and high-resolution human body models, Bioelectromagnetics, (2005).

25. Ulbrich M. and Röthlingshöfer L. and Cordes A. and Leonhardt S., Simulation of Electromagnetic Fields for Impedance Measurements in Medical Engineering, 44. Jahrestagung der Deutschen Gesellschaft für Biomedizinische Technik (BMT2010), Rostock, Deutschland, (2010).

26. Clemens M., Discrete Electromagnetism with the Finite Integration Technique, Progress In Electromagnetics Research, 65-87, (2001). http://dx.doi.org/10.2528/PIER00080103

27. Gabriel C. and Gabriel S. and Lau R.W., The dielectric properties of biological tissues: II Measurements in the frequency range 10 $\mathrm{Hz}$ to $20 \mathrm{GHz}$, in Physics in Medicine and Biology, 41, (1996). http://dx.doi.org/10.1088/0031-9155/41/11/002

28. Xitron Technologies, Hydra ECF/ICF (Model 4200), Bioimpedance spectrum analyser. Operating manual, Xitron Technologies Inc., San Diego, (2001).

29. Hanai T., Electrical properties of emulsions in Sherman DH, ed. Emulsions Science, London Academic, 354-477, (1968).

30. Matthie J., Second generation mixture theory equation for estimating intracellular water using bioimpedance spectroscopy, J Appl Physiol, 99, 780-781, (2005). http://dx.doi.org/10.1152/japplphysiol.00145.2005

31. Krämer M., A new model for the determination of fluid status and body composition from bioimpedance measurements, Physiological Measurement, 27, 901-919, (2006). http://dx.doi.org/10.1088/0967-3334/27/9/011

32. National Library of Medicine, "The visible human project." [Online]. Available: http://www.nlm.nih.gov/research/visible/

33. Medrano G. and Eitner F.and Floege J.and Leonhardt S., A Novel Bioimpedance Technique to Monitor Fluid Volume State During Hemodialysis Treatment, ASAIO J., 56(3), 215-20, (2010). http://dx.doi.org/10.1097/MAT.0b013e3181d89160 